\title{
Subclavian vein puncture-induced massive pulmonary hemorrhage and hemoptysis during pacemaker implantation
}

\author{
Nizamettin Selcuk Yelgec, Altug Osken, Ceyhan Turkkan, Ahmet Taha Alper \\ Department of Cardiology, Siyami Ersek Thoracic and Cardiovascular Surgery Center, Training and Research Hospital, Istanbul, Turkey
}

\begin{abstract}
We present a rare and interesting case of subclavian vein puncture-induced focal intraparenchymal lung hemorrhage and massive hemoptysis developed during CRT-D implantation. Clinical picture advanced to pulmonary edema in seconds. A noncontrast multiple-detector computed tomography scan revealed focal alveolar hemorrhage in the lung tissue right under the pacemaker pocket, but remarkably, there was no pneumothorax. This case shows that if cough and hemoptysis suddenly develop during subclavian puncture, injury of the adjoining lung because of parenchymal puncture should be considered as a complication.
\end{abstract}

Keywords: Hemoptysis; pacemaker; pulmonary hemorrhage.

Cite this article as: Yelgec NS, Osken A, Turkkan C, Alper AT. Subclavian vein puncture-induced massive pulmonary hemorrhage and hemoptysis during pacemaker implantation. North Clin Istanb 2018;5(3):254-255.

$\mathrm{P}$ ermanent pacemaker implantation remains one of the core skills of cardiologists. Implantation of a pacemaker lead via subclavian puncture carries a small but significant risk of access-related complications. Although hemoptysis related to the injury of lung parenchyma is a rare complication of subclavian vein access and is usually self-limited, it can affect prognosis in critically ill patients.

We report a case of hemoptysis occurring during the vein puncture for cardiac resynchronization therapy defibrillator (CRT-D) implantation.

\section{CASE REPORT}

A 59-year-old patient who had had a coronary artery bypass graft and prosthetic mitral valve replacement 5 years ago was admitted to our hospital because of bradycardia-induced syncope. His electrocardiogram showed A-V dissociation, $3^{\text {rd }}$ degree A-V block, and left bundle branch block with QRS greater than $150 \mathrm{msn}$. His escape rhythm was about 40 beats per minute with a nor- mal sinus node activity. Echocardiographic examination revealed diffuse hypokinesis and an ejection fraction of about 35\%. His New York Heart Association functional class was III under optimal medical therapy. A decision to implant a CRT-D pacemaker was taken. He was under warfarin treatment because of the mechanical valve, and his INR was 1.7 on the day of the operation.

After the patient was prepared for surgery, a leftarm contrast venography was obtained to be used as a roadmap for the venous puncture. This was the preferred method for venous access in our department. The first puncture of the atrial lead was accomplished successfully. While trying for the second puncture for ventricular lead, the patient began to cough. At the beginning, it seemed an unimportant nonspecific cough. But a few minutes later, the patient started to have massive hemoptysis and dyspnea and became cyanotic, orthopneic, and anxious. Fluoroscopy showed no signs of pneumothorax. The clinical picture was compatible with an acute pulmonary edema. We intervened with oxygen, diuretics, morphine, and head elevation. The patient became confused and agitated and tried to sit on the operation table. The im-

Received: May 15, 2017 Accepted: December 11, 2017 Online: May 29, 2018

Correspondence: Dr. Altug OSKEN. Siyami Ersek Gogus Kalp ve Damar Cerrahisi Egitim ve Arastirma Hastanesi, Kardiyoloji Klinigi, Istanbul, Turkey.

Phone: +90 2165424444 e-mail: alosk@hotmail.com

(c) Copyright 2018 by Istanbul Provincial Directorate of Health - Available online at www.northclinist.com 
plantation procedure was stopped as it was impossible to continue. We removed the wires and quickly closed the pocket. The patient was then managed in the intensive care unit. Multiple-detector computed tomography (MDCT) scans revealed no pneumothorax but localized pulmonary hemorrhage in the lung parenchyma under the newly fashioned pacemaker pocket. On noncontrast scans, there was hyperdense airspace consolidation in the left upper lung parenchyma within the alveolar space (Fig. 1).

After medical therapy for respiratory distress and stabilization of heart failure, CRT-D was implanted uneventfully, and the patient was discharged without any problem.

\section{DISCUSSION}

Subclavian vein puncture is the most frequently practiced approach for pacemaker lead insertion because it is simple and suitable for multiple-lead insertion [1]. However, the debate regarding the safety and efficacy of this method remains unresolved. The possible shortterm complications of this approach are pneumothorax, hemothorax, and lung parenchymal puncture [1]. If the cephalic subclavian vein or cephalic vein access is difficult, there is a risk of the adjoining lung being accidentally punctured during the procedure. Contrast venography might help to localize the subclavian vein. Alternatively, the use of wire-guided venous access through a superficial brachial vein, either by the cephalic or basilic vein has been suggested [2]. The wire is pushed into the subclavian vein, and so subclavian puncture under fluoroscopy is made easier and safer.

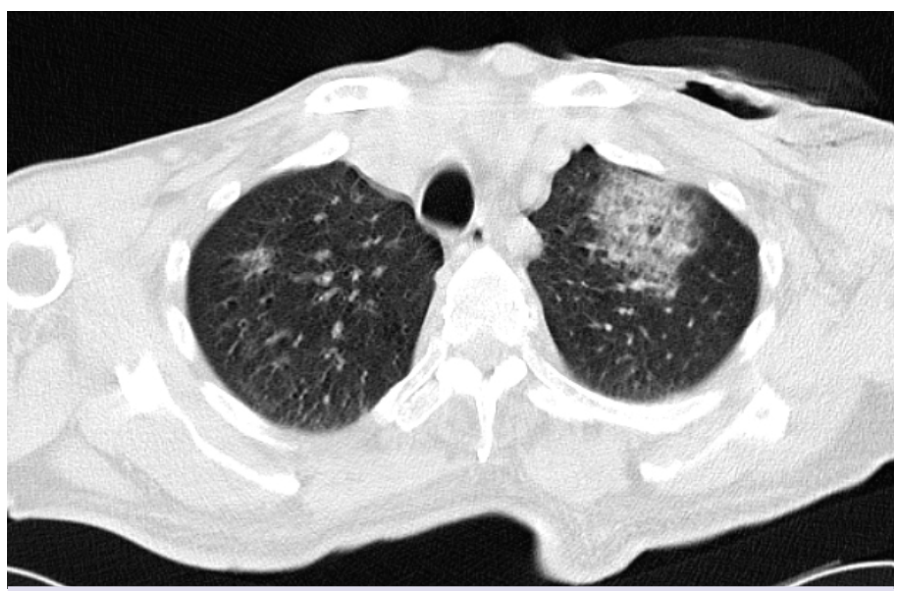

FIGURE 1. Noncontrast chest CT revealing localized alveolar hemorrhage under newly fashioned pacemaker pocket on the left without pneumothorax.
Although there is $1 \%-3 \%$ risk of pneumothorax and hemothorax, a literature review revealed that the risk of subclavian vein puncture-induced parenchymal injury resulting in pulmonary hemorrhage without pneumothorax is extremely rare during subclavian puncture [2-3]. The outcome is usually benign, but it can be more lethal in patients with heart failure, chronic lung disease, or coagulopathy. The patient's high INR on the day of operation probably facilitated the occurrence of the massive pulmonary hemorrhage. Additionally, the presence of serious heart failure caused serious respiratory distress and finally pulmonary edema.

\section{CONCLUSION}

Possible access-related complications during pacemaker implantation include pneumothorax, hemopneumothorax, lung laceration, inadvertent arterial puncture, air embolism, arteriovenous fistula, thoracic duct injury, and brachial plexus injury. In patients with respiratory disease, heart failure and coagulopathy some of these complications might cause severe respiratory distress. An absence of pneumothorax does not eliminate the possibility of lung injury during pacemaker insertion. Patients with respiratory distress after pacemaker implantation who do not show any pneumothorax on initial chest $\mathrm{x}$-ray should be further evaluated using MDCT.

Informed Consent: Written informed consent was obtained from the patient who participated in this study.

Conflict of Interest: No conflict of interest was declared by the authors.

Financial Disclosure: The authors declared that this study has received no financial support.

Authorship Contributions: Concept - N.S.Y., A.O.; Design N.S.Y.; Supervision - N.S.Y., A.O.; Materials - N.S.Y., A.O.; Data collection \&/or processing - A.O., C.T.; Analysis and/or interpretation - N.S.Y., A.O.; Writing - N.S.Y.; Critical review - C.T., A.T.A.

\section{REFERENCES}

1. Littleford PO, Parsonnet V, Spector SD. Method for the rapid and atraumatic insertion of permanent endocardial pacemaker electrodes through the subclavian vein. Am J Cardiol 1979;43:980-2. [CrossRef]

2. Kossaify A, Nicolas N, Edde P. Hemoptysis after subclavian vein puncture for pacemaker implantation: importance of wire-guided venous puncture. Clin Med Insights Case Rep 2012;5:119-22. [CrossRef]

3. Goldberg A, Rosenfeld I, Marmor A. Hemoptysis-a rare complication of pacemaker implantation. Indian Pacing Electrophysiol J 2008;8:756. 